Rev. Inst. Flor. v. 26 n. 2 p. 137-145 dez. 2014

http://dx.doi.org/10.4322/rif.2014.010

ISSN impresso 0103-2674/on-line 2178-5031

\title{
PINUS TROPICAL COM POTENCIAL PARA USO EM PLANTIOS COMERCIAIS NO BRASIL ${ }^{1}$ TROPICAL PINE FOR COMMERCIAL PLANTING IN BRAZIL
}

\author{
Marcos Silveira WREGE ${ }^{2,5}$; Elenice FRITZSONS'; \\ Jarbas Yukio SHIMIZU ${ }^{3}$; Ananda Virgínia de AGUIAR ${ }^{2}$; Paulo Henrique CARAMORI ${ }^{4}$
}

RESUMO - Entre os pinus tropicais, Pinus caribaea var. hondurensis é uma variedade natural, oriunda de região com clima tropical na América Central. A espécie se aclimatou bem em várias partes do mundo, em regiões com alta pluviosidade e temperaturas elevadas. Embora não tolere geadas severas, pode ser plantada em partes da região Sul do Brasil onde o clima é mais quente e a frequência de geadas é menor e é indicado para plantio em boa parte do Brasil, em mais de 3,5 milhões de $\mathrm{km}^{2}$. Essa é uma variedade que apresenta alto rendimento em madeira de boa qualidade, além de possibilitar a exploração da resina. Neste trabalho, são apresentadas as regiões no Brasil que apresentam potencial para cultivo dessa variedade em regime de silvicultura intensiva, tomando como base sua região de origem no mundo. São indicadas para plantio comercial as zonas com os menores riscos de geada nos estados da região Sul, no Estado de São Paulo e nas demais regiões que oferecem condições climáticas favoráveis, principalmente de disponibilidade hídrica para o desenvolvimento dessa variedade de pinus.

Palavras-chave: Pinus caribaea var. hondurensis; zoneamento; riscos climáticos; balanço hídrico; evapotranspiração.

\begin{abstract}
Among tropical pines, Pinus caribaea var. hondurensis is a natural variation from the tropical regions of Central America. The species has adapted well in various parts of the world, in regions with high rainfall and high temperatures. Although intolerance to severe frosts, it can be grown in parts of southern Brazil, where the climate is hot, with infrequent frosts and even can be used for commercial planting in over 3.5 million square kilometers.This variety presents high yield in quality wood as well as resin. This work shows regions in Brazil where environmental conditions are favorable for commercial plantations with this variety, by making comparisons with the conditions in its origin. Climatic zones within the Southern Region, as well as in the state of São Paulo and elsewhere with the lowest risks of frost and plenty of water for the development of this pine variety are presented.
\end{abstract}

Keywords: Pinus caribaea var. hondurensis; zoning; climate risks; water balance; evapotranspiration.

\footnotetext{
${ }^{1}$ Recebido para análise em 03.06.14. Aceito para publicação em 16.09.14.

${ }^{2} E$ mbrapa Florestas, Estrada da Ribeira, km 111, Guaraituba, 83411-000 Colombo, PR, Brasil.

${ }^{3}$ Autônomo. Pesquisador aposentado da Embrapa Florestas. shimizujy@gmail.com

${ }^{4}$ Instituto Agronômico do Paraná - IAPAR, Rodovia Celso Garcia Cid, km 375, Três Marcos, 86047-902 Londrina, PR, Brasil. pcaramori@gmail.com

${ }^{5}$ Autor para correspondência: Marcos Silveira Wrege - marcos.wrege@embrapa.br
} 


\section{INTRODUÇÃO}

Espécies de pinus vem sendo cultivadas no Brasil há mais de um século e os primeiros plantios utilizando espécies subtropicais iniciaram-se por volta de 1936, estabelecidos pelo Serviço Florestal do Estado de São Paulo, atual Instituto Florestal de São Paulo. Em meados de 1960, iniciaram-se os plantios comerciais nas regiões Sul e Sudeste, sendo que as espécies mais difundidas foram $P$. elliottii e $P$. taeda, introduzidas dos Estados Unidos e, em menor escala, os tropicais $P$. caribaea e $P$. oocarpa. Hoje, estima-se que o plantio de pinus atinja $1.562,782$ hectares no Brasil (Associação Brasileira de Produtores de Florestas Plantadas - ABRAF, 2013).

Pinus caribaea é um dos pinus mais difundidos no mundo nas regiões tropicais e apresenta três variedades: hondurensis, bahamensis e caribaea. Pinus caribaea var. hondurensis é originário da América Central, onde o clima é tipicamente tropical, com grandes variações na precipitação pluviométrica, em pequenas extensões de terra e com temperaturas médias anuais elevadas. Sua região de origem estende-se desde o nível do mar até 700 metros s.n.m. (metros sobre o nível do mar), mas pode ser encontrada, também, até 1.000 metros s.n.m. (Shimizu e Sebbenn, 2008).

O crescimento de $P$. caribaea var. hondurensis pode chegar a $30 \mathrm{~m}$ de altura, com DAP (diâmetro do tronco a 1,3 m de altura) de até $80 \mathrm{~cm}$. Em alguns casos, pode atingir até mesmo $45 \mathrm{~m}$, com DAP de $135 \mathrm{~cm}$ em condições climáticas favoráveis. De modo geral, o fuste é reto, com poucas ramificações laterais (Dvorak et al., 2000). A produção de madeira pode variar de $21 \mathrm{~m}^{3} \mathrm{ha}^{-1}$ a $43 \mathrm{~m}^{3} \mathrm{ha}^{-1}$ até o terceiro ano após o plantio. Essa é uma variedade de rápido crescimento e produtora de madeira de boa qualidade, além de produzir resina que pode ser extraída comercialmente.

As condições climáticas na região de origem de $P$. caribaea var. hondurensis são caracterizadas por temperaturas médias anuais de $22{ }^{\circ} \mathrm{C}$ a $27{ }^{\circ} \mathrm{C}$ (Dvorak et al., 2000) e pluviosidades médias anuais de $670 \mathrm{~mm}$ a $2.400 \mathrm{~mm}$. O déficit hídrico varia de $200 \mathrm{~mm}$ a $250 \mathrm{~mm}$ (Castro et al., 2010). Como a amplitude das variáveis climáticas na sua região de origem é elevada, desenvolveu a capacidade de se adaptar bem em muitas regiões do mundo. Existem raças geográficas litorâneas, bem como de regiões montanhosas e continentais. Contudo, elas não toleram ambientes onde ocorrem geadas severas.
A distribuição geográfica de $P$. caribaea var. hondurensis é mais ampla do que das outras variedades (bahamensis e caribaea), sugerindo que, provavelmente, estas tenham surgido a partir da primeira, apresentem menor versatilidade para se adaptarem e dependam de nichos mais restritos para se desenvolverem. No processo de adaptação, $P$. caribaea tornou-se capaz de tolerar o fogo rasteiro, por meio da espessa camada de casca desenvolvida no tronco, especialmente do córtex que protege o tecido cambial. As procedências costeiras apresentam especial resiliência aos efeitos dos ventos fortes e salinos, devido à evolução em ambiente litorâneo regularmente fustigado por furacões (International Center for Research in Agroforestry - ICRAF, 2013). A variedade hondurensis é a mais plantada, inclusive no Brasil, nas regiões Norte, Centro-Oeste e Sudeste (Aguiar et al., 2011). Neste trabalho, visou-se à identificação de regiões no Brasil que apresentem potencial para o uso de P. caribaea var. hondurensis em plantios comerciais com baixo risco climático. Isso inclui locais com os menores riscos de geada na região Sul.

\section{MATERIAL E MÉTODOS}

Um dos grandes problemas para o uso em plantio comercial de espécies arbóreas tropicais está no risco de ocorrência de geadas, comuns nas regiões Sul e Sudeste do Brasil, inclusive de espécies de pinus tropicais, como o Pinus caribaea var. hondurensis. Assim, neste trabalho, usou-se o risco de ocorrência de geadas como critério de delimitação de zonas para plantio dessa variedade de pinus nos estados da região Sul do Brasil e para o Estado de São Paulo, onde os riscos de geadas são comuns.

Os riscos de geada foram estabelecidos usando a série temporal de dados climáticos diários dos últimos 30 anos, fornecidos pelas instituições estaduais de pesquisa do Paraná e do Rio Grande do Sul (Instituto Agronômico do Paraná - IAPAR e Fundação Estadual de Pesquisa Agropecuária FEPAGRO). Foram usados dados de temperatura mínima do ar e foram calculadas as frequências com que a temperatura chega a menos de $3{ }^{\circ} \mathrm{C}$ no mês mais frio do ano (julho). A frequência foi calculada da seguinte maneira (Equação 1):

frequência de geadas $=(\mathrm{n} / 10) \times 100 \quad($ Equação 1$) ;$ 
em que:

n: número de dias, a cada 10 dias, em que a temperatura mínima do ar atinge menos de $3{ }^{\circ} \mathrm{C}$ no abrigo meteorológico.

Foi usada a temperatura mínima do ar de $3{ }^{\circ} \mathrm{C}$ no abrigo meteorológico (situado a 1,5 metro de altura), considerando-se que a mesma está relacionada à ocorrência de geadas devido ao fenômeno de inversão térmica que ocorre em noites estáveis de inverno, ocasionando um gradiente médio de temperatura, entre o abrigo e a relva, superior a $4{ }^{\circ} \mathrm{C}$. Essa diferença entre a medição feita no abrigo e a relva pode variar entre $3,3{ }^{\circ} \mathrm{C}$ a $7{ }^{\circ} \mathrm{C}$ (Silva e Sentelhas, 2001; Grodzki et al., 1996).

Por meio de análise estatística multivariada, os riscos de geadas foram calculados e correlacionados com o modelo de superfície do terreno (altitude) e, posteriormente, aos modelos de latitude e de longitude, formando uma equação de regressão linear múltipla, usada em sistema de informações geográficas para mapear os riscos de ocorrência de geadas (Wrege et al., 2011) (Equação 2):

risco de geada $\left(\begin{array}{lll}0 & \text { a } & 1\end{array}\right)=\alpha+\beta \mathrm{x}$ latitude + $\chi \mathrm{x}$ longitude $+\delta \mathrm{x}$ altitude

(Equação 2);

em que,

$\alpha=$ constante;

$\beta=$ coeficiente da latitude (representada em graus decimais negativos);

$\chi=$ coeficiente da longitude (representada em graus decimais negativos);

$\delta=$ coeficiente da altitude (metros).
Os valores de $\alpha, \beta, \chi$ e $\delta$ são apresentados na Tabela 1.

A classificação quanto aos riscos de geadas para as regiões Sul e Sudeste foi feita da seguinte maneira: 1. estados de Minas Gerais, São Paulo e Paraná: a zona foi considerada favorável quando os riscos de geada encontravam-se classificados abaixo de $20 \%$; zona marginal, quando os riscos foram classificados entre 20-30\%, e zona desfavorável, quando os riscos eram superiores a $30 \%$; 2. para Santa Catarina e Rio Grande do Sul, mudou-se a classificação para: zona favorável, quando os riscos foram classificados abaixo de $10 \%$, e zona desfavorável, quando os riscos eram superiores a $10 \%$. Neste último caso, não foi utilizada zona marginal, devido às características desses estados da região Sul, sujeitos à ocorrência de geadas mais fortes, isto é, geadas associadas à ocorrência de temperaturas mínimas absolutas do ar menores e com maior frequência, que inviabilizam o cultivo de $P$. caribaea var. hondurensis.

O modelo de superfície do terreno usado foi o GTOPO30, o qual reproduz as cotas altimétricas do Brasil, elaborado pelo serviço geológico dos Estados Unidos (United States Geological Survey - USGS, 2011) a partir de imagens de satélite da Terra. Foram elaborados, também, modelos dos paralelos e dos meridianos (representando o efeito de continentalidade), modelos que reproduzem a latitude e a longitude do Brasil, na escala 1:250.000, como o modelo de superfície do terreno.

Tabela 1. Coeficientes da equação de regressão linear múltipla dos riscos de geada para os estados de São Paulo, Paraná, Santa Catarina e Rio Grande do Sul (Wrege et al., 2005).

Table 1. Coefficients of the multiple linear regression of frost risk in the states of São Paulo, Paraná, Santa Catarina and Rio Grande do Sul (Wrege et al., 2005).

\begin{tabular}{lcccc}
\hline Estado & \multicolumn{4}{c}{ Coeficientes da equação de regressão } \\
\cline { 2 - 5 } & $\alpha$ & $\beta$ & $\chi$ & $\delta$ \\
\hline $\begin{array}{l}\text { Estados de São Paulo } \\
\text { e Paraná }\end{array}$ & $-2,8864$ & $-0,1081$ & $-0,004937$ & 0,0005165 \\
$\begin{array}{l}\text { Estados de Santa Catarina } \\
\text { e Rio Grande do Sul }\end{array}$ & $-2,6701$ & $-0,09784$ & 0,001844 & 0,0004057 \\
\hline
\end{tabular}


Para as demais regiões do Brasil, pelo fato das restrições climáticas serem diferentes, foram adotados outros critérios. Utilizaram-se, assim, as temperaturas médias anuais e o balanço hídrico climático (P-ETP), em que $\mathrm{P}$ é o total de precipitação pluviométrica de um mês (mm) e ETP é a evapotranspiração potencial acumulada em um mês (mm), calculada pelo método de Thornthwaite (1948) (Wrege et al., 2011), pelas seguintes fórmulas (Equações 3 a 6):

$\mathrm{ETP}=\mathrm{f} \times 16 \times(10 \times \mathrm{tmed} / \mathrm{I}) ;^{\mathrm{a}} \quad$ Equação 3; em que:

$\mathrm{f}=[0,006 \times(-1) \times$ latitude $+1,01] \quad($ Equação 4);

$\mathrm{I}=\sum_{12}^{1}(\text { tmed } / 5)^{1,514}$

(Equação 5);

$\mathrm{a}=6,75 \times 10^{-7} \times \mathrm{I} 3-7,71 \times 10^{-5} \times \mathrm{I}^{2}+0,01791 \times$

$\mathrm{I}+0,492$

(Equação 6);

em que:

f: representa a função de ajuste do modelo para cada local, considerando-se que a equação é dada para calcular a ETP em um período de 12 horas de brilho solar, o que não ocorre em todas as regiões;

I: é o índice anual de calor;

a: é a função cúbica do índice anual de calor;

tmed: é a temperatura média do ar nos meses de janeiro a dezembro, em ${ }^{\circ} \mathrm{C}$;

$\sum_{12}^{1}$ tmed: é o somatório das temperaturas médias do ar de janeiro a dezembro, em ${ }^{\circ} \mathrm{C}$.

A latitude, na equação, foi expressa em graus decimais negativos e serviu para calibrar os valores de ETP para cada local.
Foram somadas as diferenças de P-ETP de cada mês para cada local, totalizando o valor de P-ETP para um ano completo. Quando o valor final foi superior a $250 \mathrm{~mm}$, o local foi considerado favorável para plantio comercial de $P$. caribaea var. hondurensis; quando se situava entre $0-250 \mathrm{~mm}$, indicou que o local foi considerado marginal e, quando os valores foram negativos, isto é, inferiores a $0 \mathrm{~mm}$, foi considerado indicativo de condição desfavorável ao plantio comercial.

A temperatura média anual do ar foi calculada usando-se as médias entre as mínimas e as máximas diárias. Essas temperaturas e a pluviosidade foram calculadas para todo o Brasil por Hamada et al. (2008). A temperatura maior que $22{ }^{\circ} \mathrm{C}$ indica zonas preferenciais, ou entre 21 e $22{ }^{\circ} \mathrm{C}$ indica zonas marginais, e menor que $21{ }^{\circ} \mathrm{C}$ foi considerada desfavorável ao desenvolvimento de $P$. caribaea var. hondurensis.

Os coeficientes das regressões usadas para o mapeamento da temperatura média anual do ar são apresentados na Tabela 2, sendo feita da mesma forma que o risco de geada, por meio de análise estatística multivariada, em que as temperaturas foram correlacionadas com o modelo de superfície do terreno (altitude), da latitude e da longitude, formando uma equação de regressão linear múltipla, usada em sistema de informações geográficas (Wrege et al., 2011).

O mapa do balanço hídrico climático foi elaborado usando-se geoestatística, por meio de krigagem por indicação, em que são relacionados os pontos dos locais de ocorrência de cada uma das estações pertencentes à rede de estações meteorológicas com os pontos das estações vizinhas.

Tabela 2. Coeficientes das equações de regressão linear múltipla das médias anuais das temperaturas mínimas e máximas do $\operatorname{ar}\left({ }^{\circ} \mathrm{C}\right)$ no Brasil (Wrege et al., 2011).

Table 2.Coefficients of multiple linear regression of the annual minimum and maximum temperature $\left({ }^{\circ} \mathrm{C}\right)$ in Brazil (Wrege et al., 2011).

\begin{tabular}{lccccc}
\hline Brasil & \multicolumn{4}{c}{ Coeficientes da equação de regressão } \\
\cline { 3 - 6 } & & $\alpha$ & $\beta$ & $\chi$ & $\delta$ \\
\hline Coeficientes da & Temp. mín. & 21,0143 & 0,107068 & $-0,01347$ & $-0,00444$ \\
equação & Temp. máx. & 29,9644 & 0,047187 & $-0,03094$ & $-0,00352$ \\
\hline
\end{tabular}


Os mapas dos limites estaduais e do limite federal utilizados foram os do Instituto Brasileiro de Geografia e Estatística - IBGE (2001), que são os mapas oficiais do Brasil.

Os mapas foram classificados delimitando-os em zonas com os limites de riscos de geadas, de temperatura e de disponibilidade hídrica necessários ao desenvolvimento de P. caribaea var. hondurensis, citados anteriormente neste tópico.

\section{RESULTADOS E DISCUSSÃO}

Atualmente, existem poucas informações de orientação sobre as melhores regiões para plantio comercial de $P$. caribaea var. hondurensis no Brasil, e são exceções os zoneamentos para o estado do Paraná (Fritzsons et al., 2013) e para o Estado do Espírito Santo (Castro et al., 2010). Anteriormente, foram feitos zoneamentos para espécies florestais, incluindo essa variedade para o Paraná e Santa Catarina (Empresa Brasileira de Pesquisa Agropecuária - EMBRAPA, 1986; 1988). Golfari e Caser (1977), também fez um zoneamento utilizando metodologia diferente, em função de não existirem séries temporais históricas de clima na época em que o trabalho foi realizado.

Os fatores limitantes para o $P$. caribaea var. hondurensis são a geada e o déficit hídrico. Estas mesmas condições foram utilizadas nos zoneamentos realizados por Golfari e Caser (1977) e por Castro et al. (2010). P. caribaea var. hondurensis tem potencial para ser cultivado em sistema intensivo em boa parte do Brasil (figuras 1 e 2), exceto nas regiões de ocorrência de geadas, como nas zonas de altitude das regiões Sul e Sudeste e onde o déficit hídrico é acentuado, como é o caso do Semiárido do Agreste. Estima-se que a área potencial de plantio comercial de $P$. caribaea var. hondurensis seja de pouco mais de 3,5 milhões $\mathrm{km}^{2}$, representando mais de $40 \%$ da área total do Brasil, que é de aproximadamente 8,5 milhões de $\mathrm{km}^{2}$.

No Litoral da região Sul, nos estados do Paraná e de Santa Catarina, o clima é tropical, com elevadas temperaturas e pluviosidade, semelhante à região de origem de algumas populações de $P$. caribaea var. hondurensis (Dvorak et al., 2000), sendo, portanto, uma região recomendada para o plantio comercial.

No Norte do paralelo $23^{\circ} 27^{\prime} \mathrm{S}$, ocorre a transição do clima temperado para o tropical, dependendo também do efeito do relevo sobre a temperatura do ar. A cada 100 metros de elevação, ocorre uma redução, em média, de $0,8{ }^{\circ} \mathrm{C}$ na temperatura do ar no Estado do Paraná (Fritzsons et al., 2008). Desse modo, o risco de geadas é maior nas zonas de maior altitude. Os riscos de geada são maiores ( $>30 \%$ ) nas seguintes condições: no Estado de São Paulo, a mais de 700 metros; no Estado do Paraná, a mais de 600 metros; em Santa Catarina, a mais de 500 metros; e no Rio Grande do Sul, a mais de 400 metros (Wrege et al., 2011). A zona de clima temperado fica, basicamente, acima dessas altitudes. Portanto, espécies suscetíveis à geada, como P. caribaea var. hondurensis, podem, a princípio, ser plantadas nos estados da região Sul e de São Paulo, em locais com altitudes inferiores às mencionadas. No entanto, ainda assim existem riscos de ocorrência de geadas esporádicas e fora de época nas zonas de altitude inferior às mencionadas, e o plantio, em muitos casos, deve ser evitado, principalmente onde o microclima for desfavorável (baixadas onde ocorre acúmulo de ar frio, topos de morros, onde as plantas ficam expostas aos ventos fortes e frios vindos do Sul, etc.).

O Litoral do Estado de São Paulo é também propício ao desenvolvimento de $P$. caribaea var. hondurensis, enquanto na região centro-oeste do estado, apesar de a temperatura do ar ser mais elevada e o risco de geadas menor $(<20 \%)$ do que no restante do estado, é classificada como marginal para o cultivo, por ser uma região de transição do Cerrado para a região Sudeste, com menor disponibilidade hídrica. Nas zonas com altitudes maiores que 700 metros, o risco de geadas é maior (> 30\%), tornando a silvicultura intensiva uma atividade de risco para $P$. caribaea var. hondurensis. 

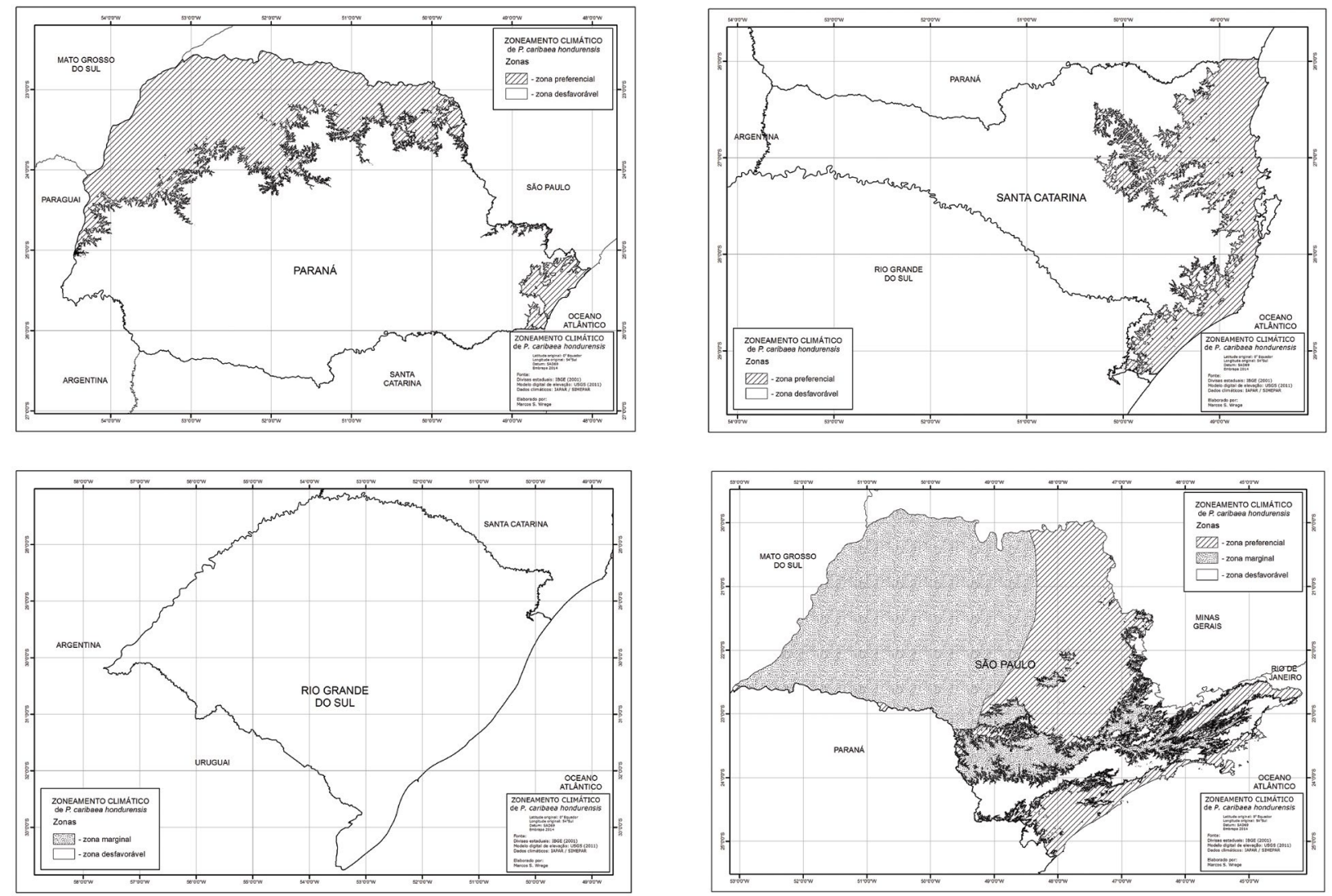

Figura 1. Delimitação de zonas com potencial para plantios comerciais de P. caribaea var. hondurensis nos estados do Paraná (a), Santa Catarina (b), Rio Grande do Sul (c) e São Paulo (d).

Figure 1. Delimitation of potential areas for commercial plantations of P. caribaea var. hondurensis in the states of Paraná (a), Santa Catarina (b), Rio Grande do Sul (c) and São Paulo (d).

O sudoeste do Paraná, oeste de Santa Catarina e noroeste do Rio Grande do Sul são as zonas com as maiores pluviosidades médias da região Sul, chegando a quase $2.000 \mathrm{~mm}$ acumulados em um ano, na média (Wrege et al., 2011). Em Santa Catarina, as áreas com elevada pluviosidade coincidem com aquelas onde as temperaturas são maiores, embora sujeitas a geadas fracas, isto é, geadas relacionadas com temperaturas do ar mais elevadas e menos frequentes, mas de baixo risco, e onde é indicado o plantio da variedade. No Rio Grande do Sul, embora o Vale do Uruguai seja uma zona de clima quente e de pluviosidade elevada, não é recomendado o plantio comercial, pois ocorrem geadas esporádicas que podem limitar o desenvolvimento. No Paraná, os maiores volumes de chuva ocorrem no sudoeste do Estado, devido à entrada das frentes frias que trazem as massas úmidas de ar e aumentam os riscos de geada, inclusive nas áreas de menor altitude, como na Foz do Rio Iguaçu. Nessa região do estado, a variação climática é acentuada em decorrência do relevo acidentado, existindo zonas com clima quente próximas de zonas com clima frio, não sendo indicado, portanto, o plantio.

As zonas indicadas neste trabalho (Figura 2), para cultivo comercial no Estado do Paraná, coincidem com as zonas recomendadas no zoneamento para espécies florestais feito em 1986 pela EMBRAPA (1986), que são as regiões bioclimáticas 4 (Planalto Norte do Paraná), 5 (Vale do Ribeira), 6 (Extremo Norte do Paraná) e 7 (Litoral). A região 3 (Oeste do Planalto de Guarapuava) não foi recomendada para plantio comercial neste trabalho, em virtude do elevado risco de geadas. 

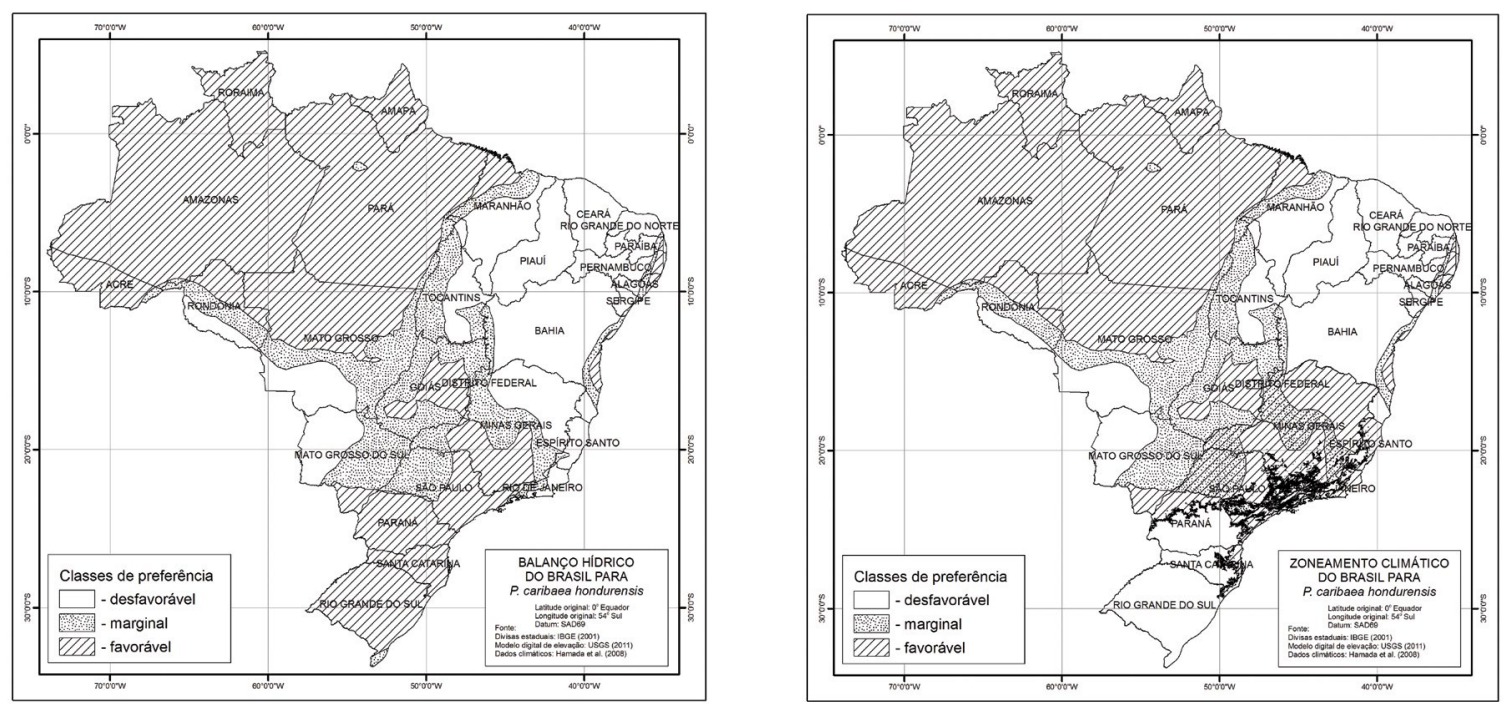

Figura 2. Classificação das zonas de balanço hídrico (a) e delimitação de zonas com potencial para plantio comercial de P. caribaea var. hondurensis no Brasil (b).

Figure 2. Classification of water balance areas (a) and delineation of potential areas for commercial planting of P. caribaea var. hondurensis in Brazil (b).

Nas zonas marginais, onde os riscos de geada são de até $20 \%$, deve-se evitar o plantio, principalmente nos estados de Santa Catarina e Rio Grande do Sul, pois nestes locais ocorre, na média, mais de uma geada forte a cada cinco anos. Nas zonas preferenciais, o risco diminui para $10 \%$, onde, em média, pode ocorrer uma geada forte a cada 10 anos. Deve-se considerar que sempre existe risco de ocorrerem geadas nas regiões Sul e Sudeste do Brasil, ainda que em níveis baixos, abaixo de $10 \%$.

Para reduzir os riscos de geada, deve-se realizar o transplantio preferentemente após o período crítico de ocorrência de geadas. De modo geral, esse período se estende entre maio e setembro na região Sul e de maio a agosto na região Sudeste. Deve-se considerar que tanto a ocorrência de geadas quanto a de estiagens é menor nas zonas litorâneas do Paraná e de Santa Catarina.

Nas regiões tropicais do Brasil, a temperatura do ar é elevada e o risco de geadas é baixo, embora o Estado de Mato Grosso do Sul esteja sujeito à entrada de sistemas frontais mais intensos que no interior paulista. O Semiárido, no Agreste Nordestino, é a região que menos se assemelha à região de origem de $P$. caribaea var. hondurensis, por causa do regime pluviométrico e do menor volume de chuvas. Na região Nordeste, também existe uma grande variabilidade climática, ocorrendo desde zonas com reduzidos volumes totais de chuva, como no Semiárido (em torno de 600 a $800 \mathrm{~mm} \mathrm{ano}^{-1}$ ), até zonas com índices elevados, como na Zona da Mata $\left(>1.500 \mathrm{~mm}^{2} \mathrm{ano}^{-1}\right)$ (Kousky e Chu, 1978). O limite crítico para o desenvolvimento de $P$. caribaea var. hondurensis é de $670 \mathrm{~mm}$ ano $^{-1}$ (menor média de pluviosidade acumulada em um ano que ocorre na região de origem deste pinus). O Litoral e o noroeste do Maranhão são zonas recomendadas para o plantio onde, na região Nordeste, são os locais em que as condições climáticas são atendidas (Hastenrath e Heller, 1977). As zonas recomendadas para o plantio são semelhantes ao zoneamento feito por Golfari e Caser (1977).

$\mathrm{Na}$ região Centro-Oeste, onde predomina o Cerrado, ocorre um período seco característico no inverno, que pode durar mais de quatro meses e interferir na produtividade do pinus. No entanto, a temperatura do ar é favorável ao desenvolvimento. Esta região é considerada marginal, por causa do balanço hídrico, que dificulta o desenvolvimento, com a possibilidade da produtividade ser menor que em outras regiões, mas por ser uma espécie perene, não o impede, exceto no primeiro ano, quando podem ser necessárias regas para o estabelecimento dos plantios, dependendo da época do plantio. 
A região Norte do país é a que mais se assemelha à região de origem do pinus tropical, principalmente em relação ao regime pluviométrico. Apesar disso, na Amazônia também existem variações do clima, incluindo o regime de chuvas, em que podem ocorrer estiagens prejudiciais à produção agrícola e florestal, sendo que estas estiagens têm se tornado mais comuns nos últimos anos (Saatchia et al., 2012). Existem referências de plantios feitos no passado, em larga escala, na região do Jarí, Estado do Pará (Golfari et al., 1978).

O zoneamento feito está de acordo com outros trabalhos realizados anteriormente (Golfari e Caser, 1977), com muitos pontos em comum, principalmente no balanço hídrico. As temperaturas utilizadas são diferentes, mas os resultados similares, pois, atualmente, conta-se com uma base de dados climáticos mais ampla, com séries temporais com mais de 30 anos de registros e o país é coberto por uma rede de estações meteorológicas com uma melhor resolução espacial. Neste trabalho, foi utilizado sistema de informações geográficas, com o uso de técnicas modernas de geoprocessamento, empregando-se nos mapeamentos regressões lineares múltiplas e geoestatística.

As várias procedências de $P$. caribaea var. hondurensis comportam-se de forma diferente conforme o ambiente e algumas se adaptam melhor que outras em cada região. A escolha da correta procedência é fundamental para o êxito dos plantios e deve ser feita de acordo com o clima e os solos de cada local. Contudo, estudos desse tipo ainda não foram feitos para essa variedade de Pinus.

Este zoneamento teve como base o estudo de regiões consideradas ótimas para o plantio considerando-se o aspecto climático, visando dar orientações sobre o estabelecimento de plantios comerciais de $P$. caribaea var. hondurensis. No entanto, é importante alertar que o êxito dos plantios comerciais de pinus não depende somente das boas condições climáticas, mas também das boas condições de solos.

\section{CONCLUSÕES}

Pinus caribaea var. hondurensis pode ser usado para plantios comerciais em regime de silvicultura intensiva em pouco mais de $40 \%$ do território brasileiro, incluindo as regiões Nordeste e Sul.
Existem, porém, regiões mais apropriadas que outras para esse fim. São locais onde não só o clima é mais quente, com menores riscos de ocorrência de geada, mas também onde as estiagens são menos frequentes.

$\mathrm{Na}$ região Sul do Brasil, existem zonas mais propícias para a silvicultura intensiva com P. caribaea var. hondurensis, sendo que as zonas marginais, em termos climáticos, que são caracterizadas por baixadas onde ocorre acúmulo de ar frio no inverno, os corredores de ventos frios e os topos de morro que sofrem a ação de ventos fortes vindos do Sul, devem ser usadas com cautela, devido aos riscos climáticos. Em um primeiro momento, tais locais podem ser usados para fins de experimentação ou no máximo para plantios concentrados nas faces norte, nordeste ou noroeste da propriedade.

Como existem diversas procedências, o sucesso da atividade está muito relacionado à escolha da procedência mais bem adaptada a cada local, onde pode ter maior desenvolvimento em função das características do clima e dos solos.

\section{REFERÊNCIAS BIBLIOGRÁFICAS}

AGUIAR, A.V.; SOUSA, V.A.; SHIMIZU, J.Y. Sistemas de produção: cultivo do Pinus. Espécies: Pinus caribaea. Disponível em: $<$ http://sistemasdeproducao.cnptia.embrapa.br/ FontesHTML/Pinus/CultivodoPinus_2ed/index. htm>. Acesso em: 14 jun. 2011.

ASSOCIAÇÃO BRASILEIRA DE PRODUTORES DE FLORESTAS PLANTADAS - ABRAF. Anuário estatístico da ABRAF 2013 ano base 2012. Brasília, DF, 2013. 145 p.

CASTRO, F. et al. Zoneamento agroclimático para espécies do gênero Pinus no estado do Espírito Santo. Floresta,v. 40, n. 1, p. 235-250, 2010. Disponível em: <http://ojs.c3sl.ufpr.br/ ojs2/index.php/floresta/article/view/17114/11274>. Acesso em: 14 maio 2013.

DVORAK, W. et al. Pinus caribaea hondurensis. In: CONSERVATION and testing of tropical and subtropical forest tree species by the CAMCORE Cooperative. Raleigh: College of Natural Resources, 2000. 234 p. 
EMPRESA BRASILEIRA DE PESQUISA AGROPECUÁRIA - EMBRAPA. Zoneamento ecológico para plantios florestais no Estado do Paraná. Brasília, DF: EMBRAPA-DDT; Curitiba: EMBRAPA-CNPF, 1986. 89 p. (Embrapa, Documentos 17).

Zoneamento ecológico para plantios florestais no Estado de Santa Catarina. Curitiba: EMBRAPA-CNPF, 1988. 113 p. (Embrapa, Documentos 21).

FRITZSONS, E.; MANTOVANI, L.E.; AGUIAR, A.V. de. Relação entre altitude e temperatura: uma contribuição ao zoneamento climático no estado do Paraná. Revista de Estudos Ambientais, v, 10, n. 1, p. 49-64, 2008. Disponível em: <http:// proxy.furb.br/ojs/index.php/rea/article/view/902>. Acesso em: 14 maio 2013.

et al. Carta de zoneamento de Pinus $\overline{c a r i b a e}$ para o estado do Paraná. Colombo: Embrapa Florestas, 2013. 24 f. (Embrapa Florestas. Documentos, 254).

GOLFARI, L.; CASER, R.L. Zoneamento ecológico da região Nordeste para experimentação florestal. Belo Horizonte: Centro de Pesquisa Florestal da Região do Cerrado, 1977. 116 p. (PRODEPEF. Série Técnica, 10). ; MOURA, V.P.G. Zoneamento ecológico esquemático para reflorestamento no Brasil (2 ${ }^{\mathbf{a}}$ aproximação). Belo Horizonte: PRODEPEF, 1978. 66 p. (PRODEPEF. Série Técnica, 11).

GRODZKI, L.; CARAMORI, P.H.; BOOTSMA, A. Risco de ocorrência de geada no estado do Paraná. Revista Brasileira de Agrometeorologia, v. 4, n. 1, p. 93-99, 1996.

HAMADA, E. et al. Cenários climáticos futuros para o Brasil. In: GHINI, R.; HAMADA, E. (Org.). Mudanças climáticas: impactos sobre doenças de plantas no Brasil. Brasília, DF: Embrapa Informação Tecnológica, 2008. v. 1, p. 25-73.

HASTENRATH, S.; HELLER, L. Dynamics of climatic hazard in the northeast Brazil. Quarterly Journal of Royal Meteorological Society, v. 103, p. 77-92, 1977.

INSTITUTO BRASILEIRO DE GEOGRAFIA E ESTATÍSTICA - IBGE. Malha municipal digital do Brasil, 2001. Rio de Janeiro, 2001. 1 CD-ROM.
INTERNATIONAL CENTER FOR RESEARCH IN AGROFORESTRY - ICRAF. Disponível em: $<$ http://www.worldagroforestrycentre.org/sea/ Copyright.htm>. Acesso em: 7 maio 2013.

KOUSKY, V.E.; CHU, P.S. Fluctuations in annual rainfall for Northeast Brazil. Journal of Meteorological Society of Japan, v. 56, p. 457-465, 1978.

OLIVEIRA, L.T.; PEZZOPANE, J.E.M.; CECÍLIO, R.A. Potencial impacto das mudanças climáticas no zoneamento do Pinus no Espírito Santo. Floresta e Ambiente, v. 18, n. 1, p. 37-49, 2011.

SAATCHIA, S. et al. Persistent effects of a severe drought on Amazonian forest canopy. Stanford: Stanford University. Disponível em: <http://www. yadvindermalhi.org/uploads/1/8/7/6/18767612/ saatchi_pnas-2012-1204651110.pdf>. Acesso em: 2 maio 2014.

SHIMIZU, J.Y.; SEBBENN, A.M. Espécies de pinus na silvicultura brasileira. In: SHIMIZU, J. Y. (Ed.). Pinus na silvicultura brasileira. Colombo: Embrapa Florestas, 2008.

SILVA, J. G.; SENTELHAS, P. C. Diferença entre temperatura mínima do ar medida no abrigo e na relva e probabilidade de sua ocorrência em eventos de geadas no Estado de Santa Catarina. Revista Brasileira de Agrometeorologia, v. 9, n. 1, p. 9-15, 2001.

THORNTHWAITE, C.W. An approach toward a rational classification of climate. Geographical Review, v. 38, p. 55-94, 1948.

UNITED STATES GEOLOGICAL SURVEY - USGS. Survey National Mapping Division. Global 30 arc second elevation data. 1999. Disponível em: $<$ http://edcwww.cr.usgs.gov/landdaac/gtopo30/ gtopo30.html>. Acesso em: 10 julho 2011.

WREGE, M.S. et al. Regiões potenciais para cultivo da cana-de-açúcar no Paraná, com base na análise do risco de geadas. Revista Brasileira de Agrometeorologia, v. 13, n. 1, p. 113-122, 2005.

et al. Atlas climático da região Sul do Brasil: estados do Paraná, Santa Catarina e Rio Grande do Sul. Pelotas: Embrapa Clima Temperado, 2011. v. 1, 332 p. 